\title{
Factores asociados en la percepción del médico sobre la relación médico-paciente
}

\author{
Johnny Francisco Casanova Saldarriaga ${ }^{1, a}$
}

RESUMEN

Objetivo: Determinar los factores asociados en la percepción de la relación médico-paciente en los médicos que laboran en el Hospital Nacional Edgardo Rebagliati Martins, 2015.

Materiales y métodos: Estudio de tipo observacional, prospectivo, transversal y analítico, de diseño no experimental. El universo de estudio corresponde a todos los médicos que laboran en el Departamento de Cirugía de Cabeza y Cuello, el cual incluye los Servicios de Otorrinolaringología (15 médicos), Oftalmología (25 médicos), y Cabeza y Cuello (14 médicos). El instrumento utilizado fue una encuesta autoadministrada sobre la base de tres temas: 1) Influencia de la tecnología y la especialización en la deshumanización de la relación médico-paciente. 2) Influencia de la judicialización de la medicina. 3) Respeto y confianza del paciente. Se efectuaron 18 preguntas basadas en las tres interrogantes principales, y se recopiló información sobre los datos sociodemográficos, educativos y culturales del médico. El instrumento fue validado por prueba de expertos, con un nivel de confiabilidad de 0.92 (alfa de Cronbach). Además de la encuesta, se realizaron entrevistas personales a los médicos encuestados, con la finalidad de averiguar su opinión personal sobre su relación médico-paciente. Los datos fueron analizados con el paquete estadístico IBM SPSS. El análisis estadístico se realizó con la prueba de chi-cuadrado, con un nivel de significancia del $5 \%$.

Resultados: La investigación evaluó a 30 mẻdicos especialistas de una media etaria de 53.57 años: el $83.3 \%$ de los médicos entrevistados era de sexo masculino, el $43 \%$ pertenecía a la especialidad de oftalmología, el $36.7 \%$ era natural de Lima, el $43.3 \%$ culminó sus estudios secundarios en colegio público, el $66.7 \%$ cursó estudios de medicina en una universidad pública, el $93.3 \%$ cursó estudios de especialidad en una universidad pública, el $46.7 \%$ manifestó que su madre nació en la sierra del Perú, el 63.3\% manifestó que su padre nació en la sierra del Perú, el $87 \%$ profesaba la religión católica, el $83 \%$ manifestó haber elegido la carrera de medicina por vocación, el $90 \%$ ejercía también la actividad médica en el sector privado, el $43 \%$ participaba como voluntario en programas de apoyo social, y el $46.7 \%$ percibía la existencia de una buena relación médico-paciente.

Conclusiones: La tecnología y la especialización no contribuyen a la deshumanización del médico. El estudio de pacientes por órganos muchas veces favorece la pérdida de visión en conjunto del paciente. La medicina basada en evidencias es necesaria solo como complemento a las experiencias del médico. Los trámites burocráticos y los plazos de espera prolongados desaniman al paciente. Es importante dedicarle más tiempo al paciente en la consulta externa. La percepción de la relación médico-paciente se correlaciona fuertemente con la satisfacción del usuario y del médico.

Palabras claves: Percepción; relación médico-paciente; factores asociados (Fuente: DeSC BIREME).

\section{Factors associated with physicians' perception of physician-patient relationship}

\author{
ABSTRACT
}

Objective: To determine the factors associated in the perception of physician-patient relationship by physicians who work at the Hospital Nacional Edgardo Rebagliati Martins, 2015.

Materials and methods: An observational, prospective, cross-sectional, analytic, non-experimental study. The study population consisted of all the physicians who work at the Head and Neck Surgery Department, which includes the Otolaryngology (15 physicians), Ophthalmology (25 physicians), and Head and Neck (14 physicians) Specialty Areas. The research instrument was a self-administered survey based on three topics: 1) Influence of technology and specialization in dehumanizing physician-patient relationship. 2) Influence of judicialization of medicine. 3) Respect and confidence of patients. Eighteen questions based on the three main topics were asked, and physician's sociodemographic, educational and cultural data were collected. The instrument was validated by a team of experts, at a confidence level of 0.92 (Cronbach's Alpha score). Besides the survey, physicians underwent a personal interview, in order to find out their personal opinion about their physician-patient relationship. Data were analyzed using IBM SPSS Statistics statistical software. Statistical analysis was conducted using chi-square test, with a significance level of $5 \%$.

Results: The research assessed 30 specialty physicians with a mean age of 53.57 years: $83.3 \%$ of the interviewed physicians were male, $43 \%$ majored in ophthalmology, 36.7\% were born in Lima, 43.3\% finished high school at a public school, $66.7 \%$ studied medicine at a public university, 93.3\% pursued major studies at a public university, $46.7 \%$ stated that their mother was born in the Peruvian highlands, $63.3 \%$ stated that their father was born in the Peruvian highlands, $87 \%$ were Catholics, $83 \%$ said that they chose to study medicine by vocation, $90 \%$ practiced medicine in the private sector, $43 \%$ volunteered in social support programs for low-income people, and $46.7 \%$ felt that there was a good physician-patient relationship. Conclusions: Technology and specialization do not contribute to dehumanization of physicians. Studying patients by organs often causes to miss the perception of the patient as a whole. Evidence-based medicine is only necessary as a complement to physician's experiences. Bureaucratic processes and long waiting periods discourage the patient. It is important to assign more time to patients in the outpatient health care service. The perception of physician-patient relationship strongly correlates to the satisfaction of both patient and physician.

Keywords: Perception; physician-patient relationship; associated factors (Source: MeSH NLM).

1. Médico cirujano, especialidad en otorrinolaringología, maestro en bioética y doctor en medicina.

a. Docente de las asignaturas de anatomía humana y ética y bioética, Facultad de Medicina Humana, Universidad de San Martín de Porres. 


\section{INTRODUCCIÓN}

En la práctica de la medicina la relación entre el médico y su paciente juega un papel muy importante, siendo esencial para una asistencia médica de alta calidad en cuanto al diagnóstico, tratamiento y pronóstico de la enfermedad.

La relación médico - paciente $(\mathrm{rmp})$ es una relación interpersonal compleja que tiene connotaciones éticas, filosóficas y sociológicas de tipo profesional, y sirve de base para la buena gestión de la salud ${ }^{(1,2)}$.

Siendo la relación médico - paciente, una relación interpersonal compleja, se presentan diversas interrogantes: ¿En qué forma se llevan a cabo una relación médico-paciente?, ¿Qué factores condicionan una buena relación médico-paciente?, ¿Qué ocasiona el descontento en el médico o en el paciente?, ¿Qué factores contribuyen a una buena relación médico-paciente?, ¿Cuáles son las características del médico que intervienen en la relación médico-paciente? ${ }^{(3,4)}$.

Existen muchos trabajos acerca de la relación médicopaciente, la mayoría con un enfoque desde el punto de vista del paciente. Poco se ha tomado en cuenta la percepción y el sentir de la otra parte de esta relación (el médico), tan importante como la del paciente para lograr la empatía necesaria para la cura de la enfermedad ${ }^{(5,6)}$.

Por parte del médico debe conocer su carácter, sus debilidades, su nivel de información, su capacidad resolutiva del problema y cuando debe recurrir a otro colega. Debe tomar más en cuenta su prestigio científico y social ${ }^{(7)}$.

Las vías de comunicación son muy importantes. Esta comunicación puede ser verbal por medio de la palabra, gestos, expresiones faciales, tacto al realizar el examen y al utilizar instrumentos ${ }^{(8)}$.

Dentro de los trabajos existentes acerca de la relación médico-paciente, existen diversas conclusiones al respecto. Ramos C. ${ }^{(9)}$ en el año 2006 concluyó que el grado de instrucción ,grupo etáreo al que pertenece el médico, la concordancia del género, la concordancia del grupo etario, el género del médico, ni la continuidad del manejo médico se asocia a la calidad percibida en la relación médicopaciente. Según Fernández J. ${ }^{(10)}$ en el año 2003 al realizar su estudio en el grado de satisfacción del usuario externo en los consultorios externos del Hospital Militar Geriátrico de Lima, determinó que el usuario en promedio se encuentra medianamente satisfecho. Guix J. ${ }^{(11)}$ en su tesis doctoral demuestra que los pacientes quieren ser informados, $96.5 \%$. Consideran que el médico es la persona más adecuada para tomar decisiones, $81.2 \%$ y ven el consentimiento informado como un procedimiento exculpatorio, $67.2 \%$. Se han realizado estudios cuyo objetivo es explorar si las diferencias raciales en la relación médico paciente, contribuyen a la disparidad en la calidad del cuidado de la salud, Somnath et al ${ }^{(12)}$. La diferencia racial en la relación médico-paciente, ayudó a explicar la disparidad en lo referente al trato, pero no en el uso de los servicios de salud. Sin embargo conocemos de la importancia del trato adecuado para una buena relación médico-paciente y la consiguiente curación.

Alonso M. y Kraftchenko O. ${ }^{(13)}$, realizaron un estudio descriptivo de la comunicación médico-paciente como parte de la formación ético profesional. En él se analiza el cumplimiento de las tres funciones de la comunicación: informativa, regulativa y afectiva, y de la empatía como un mecanismo de comprensión mutua.

Los usuarios son el objeto último de la prestación de los servicios y por ello pueden aportar información sobre elementos que no es posible obtener por otros medios. A esto se le denomina la calidad percibida ${ }^{(14,15)}$. Al evaluar la calidad del servicio prestado a través de esta perspectiva el usuario es considerado como juez y elemento central en la organización sanitaria, Parasuman et al, $1985^{(15)}$, Parasuman et al $1994^{(16)}$; Biedman y Serrano, $2007^{(17)}$.

La pregunta que formula el problema de investigación es la siguiente: ¿Cuáles son los factores asociados a la percepción de la relación médico-paciente de los médicos que laboran en el Hospital Nacional Edgardo Rebagliati Martins 2015?.

La presente Investigación pretende colaborar en la explicación de respuestas positivas o negativas en cuanto a la relación médico - paciente, la cual constituye el pilar principal en la consecución de un buen diagnóstico, tratamiento y pronóstico.

Los resultados ayudarán a mejorar la relación médico paciente que constituye un factor muy importante en la curación y pronóstico del paciente. Al curarse el paciente en forma rápida y satisfactoria se ahorra dinero a la institución donde acude.

Este trabajo ha tenido como objetivo dilucidar las fortalezas y debilidades que caracterizan a la relación médico-paciente, y ante dicho análisis poder proponer mejoras para la optimización de dicha relación y la consecuente mejora de la percepción que los médicos tengan de ella.

\section{MATERIALES Y MÉTODOS}

El presente es un estudio de tipo observacional, prospectivo, transversal y analítico. El diseño efectuado corresponde al no experimental. 
Con respecto a la población, el universo de estudio corresponde a todos los médicos que laboran en el Departamento de Cirugía de Cabeza y Cuello, el cual incluye los servicios de: Otorrinolaringología (15 médicos), Oftalmología (25 médicos), Servicio de Cabeza y Cuello (14 médicos). Los criterios de selección utilizados fueron los siguientes:

\section{Criterio de Inclusión}

Médicos que deseen participar voluntariamente.

\section{Criterio de Exclusión}

Médicos con menos de 3 años en la especialidad. Se excluyeron médicos que están de vacaciones, descanso médico, de comisión de servicio o en sala de operaciones en los momentos de efectuar la encuesta y/o entrevistas.

\section{Muestra}

Se trabajó con la totalidad de médicos que cumplieron los criterios de selección.

\section{Recolección de datos e instrumentos}

La recolección de datos se efectuó directamente por el investigador.

El instrumento utilizado fue una encuesta auto administrada de 18 preguntas sobre la base de tres temas:

1. Influencia de la tecnología y la especialización en la deshumanización de la relación médico - paciente.

2. Influencia de la judicialización de la medicina.

3. Respeto y confianza del paciente.
Se obtuvieron datos sociodemográficos, educativos y culturales del médico El instrumento fue validado por prueba de expertos, nivel de confiabilidad 0.92 (alfa de cronbach).

Además de la encuesta se procedió a las entrevistas personales a médicos de las 3 especialidades escogidos al azar.

\section{Procesamiento y análisis}

Con los datos obtenidos se construyó una base de datos y se procedió a realizar análisis univariado, análisis bivariado y multivariado. Se realizó un análisis cualitativo de las implicancias de los resultados. Los datos fueron analizados con el paquete estadístico IBM SPSS Statistics 22. El análisis estadístico se realizó con la prueba de Chi cuadrado con un nivel de significancia del $5 \%$.

\section{RESULTADOS}

La investigación evaluó 30 médicos especialistas, los cuales presentan una media etarea de 53.57 años (DE 7.60) (Figura 1). El 83.3\% de los médicos entrevistados son del sexo masculino, el $43 \%$ de ellos son de la especialidad de Oftalmología, 30\% de la especialidad de Otorrinolaringología y $26.7 \%$ de la especialidad de Cirugía de Cabeza y Cuello (Tabla 1). El $36.7 \%$ son naturales de Lima, $63.3 \%$ de provincias (Tabla 2) de un nivel socioeconómico medio y $66.7 \%$ cursó estudios de medicina en una Universidad pública de Lima (Tabla 3).

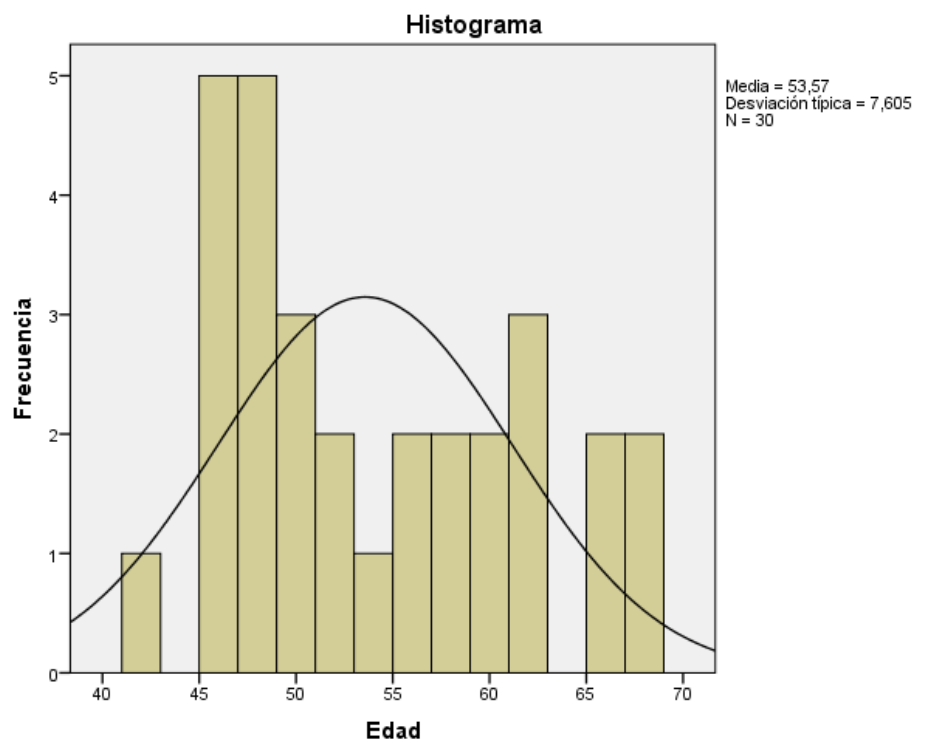

Figura 1. Distribución de los médicos según edad. Hospital Nacional Edgardo Rebagliati Martins, 2015 
Factores asociados en la percepción del médico sobre la relación médico-paciente

Tabla 1. Distribución de los médicos según especialidad médica. Hospital Nacional Edgardo Rebagliati Martins, 2015

$\begin{array}{llccc} & & \text { Frecuencia } & \text { Porcentaje } & \begin{array}{l}\text { Porcentaje } \\ \text { acumulado }\end{array} \\ \text { Válido } & \text { Otorrinolaringología } & 9 & 30,0 & 30,0 \\ & \begin{array}{l}\text { Oftalmología } \\ \text { Cirugía de cabeza y }\end{array} & 13 & 43,3 & 73,3 \\ & \text { cuello } & 8 & 26,7 & 100,0 \\ & \text { Total } & 30 & 100,0 & \end{array}$

Tabla 2. Distribución de los médicos según lugar de residencia. Hospital Nacional Edgardo Rebagliati Martins, 2015

$\begin{array}{llccc} & & \text { Frecuencia } & \text { Porcentaje } & \begin{array}{c}\text { Porcentaje } \\ \text { acumulado }\end{array} \\ \text { Válido } & \text { Lima Metropolitana } & 11 & 36,7 & 36,7 \\ & \text { Región Costa } & 7 & 23,3 & 60,0 \\ & \text { Región Sierra } & 10 & 33,3 & 93,3 \\ & \text { Región Selva } & 2 & 6,7 & 100,0\end{array}$

Tabla 3. Distribución de los médicos según universidad de procedencia. Hospital Nacional Edgardo Rebagliati Martins, 2015

$\begin{array}{llccc} & & \text { Frecuencia } & \text { Porcentaje } & \begin{array}{c}\text { Porcentaje } \\ \text { acumulado }\end{array} \\ \text { Válido } & \text { Pública en Lima } & 20 & 66,7 & 66,7 \\ & \text { Pública en provincia } & 8 & 26,7 & 93,3 \\ & \text { Privada en Lima } & 2 & 6,7 & 100,0\end{array}$

Sus padres en un $63.3 \%$ han nacido en la sierra del Perú (Tabla 4 y 5). En cuanto a la religión que profesan un $86.7 \%$ es la religión católica. Un $83.3 \%$ prefiere que la elección de la carrera de Medicina Humana es por vocación (Tabla 6). En igual porcentaje manifiestan tener al menos un hijo.
La mayoría $73.3 \%$ no tiene hijos estudiando medicina y $90 \%$ ejerce la actividad médico privada además de la actividad hospitalaria, y sólo $43.3 \%$ participa como voluntario en programas de apoyo social.

Tabla 4. Distribución de los médicos según lugar de procedencia del padre. Hospital Nacional Edgardo Rebagliati Martins, 2015

$\begin{array}{llccc} & & \text { Frecuencia } & \text { Porcentaje } & \begin{array}{c}\text { Porcentaje } \\ \text { acumulado }\end{array} \\ \text { Válido } & \text { Lima Metropolitana } & 20 & 66,7 & 66,7 \\ & \text { Región Costa } & 8 & 26,7 & 93,3 \\ & \text { Región Sierra } & 2 & 6,7 & 100,0 \\ & \text { Región Selva } & 30 & 100,0 & \\ & \text { Total } & 30 & 100,0\end{array}$


Tabla 5. Lugar de procedencia de la madre * percepción actual

\begin{tabular}{llcrcr} 
& & \multicolumn{2}{c}{ Percepción actual } & \multicolumn{2}{c}{ Total } \\
& & Buena & No Buena & \\
Lugar de procedencia & Lima & 3 & 2 & 5 \\
de la madre & Metropolitana & 3 & 6 & 9 \\
& Región Costa & 6 & 14 & 1 \\
& Región Sierra & 3 & 1 & 1 \\
& Región Selva & 0 & 1 & 30
\end{tabular}

\begin{tabular}{|c|c|c|c|}
\hline \multicolumn{4}{|c|}{ Pruebas de Chi-cuadrado } \\
\hline & Valor & $\mathrm{GI}$ & $\begin{array}{c}\text { Significación } \\
\text { asintótica (bilateral) }\end{array}$ \\
\hline $\begin{array}{l}\text { Chi-cuadrado de } \\
\text { Pearson }\end{array}$ & $3,367 a$ & 4 & ,498 \\
\hline Razón de verosimilitud & 4,147 & 4 & ,387 \\
\hline $\begin{array}{l}\text { Asociación lineal por } \\
\text { lineal }\end{array}$ & ,359 & 1 &, 549 \\
\hline $\mathrm{N}$ de casos válidos & 30 & & \\
\hline
\end{tabular}

Tabla 6. Factores asociados para la elección de la carrera de Medicina Humana

$\begin{array}{llccc} & & \text { Frecuencia } & \text { Porcentaje } & \begin{array}{r}\text { Porcentaje } \\ \text { acumulado }\end{array} \\ \text { Válido } & \text { Tradición Familiar } & 3 & 10,0 & 10,0 \\ & \begin{array}{l}\text { Por mejoras } \\ \text { económicas }\end{array} & 2 & 6,7 & 16,7 \\ & \text { Vocación } & 25 & 83,3 & 100,0 \\ & \text { Total } & 30 & 100,0 & \end{array}$

El $83.3(26 / 30)$ de los médicos entrevistados manifiesta que la elección de la carrera de medicina humana es por vocación y el 53.3\% de los médicos encuestados consideran que el desarrollo de la tecnología y la súper especialización ha generado parcialmente cierto grado de deshumanización (Tabla 7 y 8). El 70\% de los médicos encuestados están satisfechos con la práctica de su especialidad.

Tabla 7. Desarrollo de la tecnología y la superespecialización en la práctica médica

$\begin{array}{llcccc} & & \text { Frecuencia } & \text { Porcentaje } & \begin{array}{c}\text { Porcentaje } \\ \text { Pocumulado }\end{array} & \begin{array}{c}\text { Porcentaje } \\ \text { acumulado }\end{array} \\ \text { Válido } & \text { Totalmente de acuerdo } & 2 & 6,7 & 6,7 & 6,7 \\ & \text { Parcialmente de acuerdo } & 16 & 53,3 & 53,3 & 60,0 \\ & \text { Totalmente en desacuerdo } & 12 & 40,0 & 100,0 & 100,0\end{array}$


Tabla 8. Estudio del paciente por órganos según especialidades y pérdida de visión de conjunto

$\begin{array}{llcccc}\text { Válido } & \text { Frecuencia } & \text { Porcentaje } & \begin{array}{c}\text { Porcentaje } \\ \text { acumulado }\end{array} & \begin{array}{c}\text { Porcentaje } \\ \text { acumulado }\end{array} \\ & \begin{array}{l}\text { Totalmente de } \\ \text { acuerdo }\end{array} & 1 & 3,3 & 3,3 & 3,3 \\ \begin{array}{l}\text { Parcialmente de } \\ \begin{array}{l}\text { acuerdo } \\ \text { Totalmente en }\end{array}\end{array} & 16 & 53,3 & 53,3 & 60,0 \\ \text { desacuerdo } & 13 & 43,3 & 43,3 & 100,0 \\ \text { Total } & 30 & 100,0 & 100,0 & \end{array}$

Cabe mencionar que solo un $46.7 \%$ de los médicos entrevistados percibe la existencia de una buena relación médico-paciente. En el procesamiento de la base de datos, según el programa estadístico IBM SPSS, no se pudo identificar factores asociados significativos en la relación médico-paciente. Sin embargo las entrevistas personales dieron mucha información en cuanto a la percepción de los médicos acerca de la relación médico-paciente (Tabla 9).

Tabla 9. Distribución de los médicos según percepción de la relación médico/paciente. Hospital Nacional Edgardo Rebagliati Martins, 2015

$\begin{array}{clccc} & & \text { Frecuencia } & \text { Porcentaje } & \begin{array}{c}\text { Porcentaje } \\ \text { acumulado }\end{array} \\ \text { Válido } & \text { Buena } & 14 & 46,7 & 46,7 \\ & \text { Regular } & 15 & 50,0 & 96,7 \\ & \text { Mala } & 1 & 3,3 & 100,0\end{array}$

\section{DISCUSIÓN}

Es de notar que los médicos de las 3 especialidades encuestadas coincidieron que para una buena relación médico-paciente se le debe dedicar mayor tiempo al paciente en la consulta externa, ya que el paciente es una persona disminuida no solo en su salud física, sino también en la parte psicológica y quiere ser escuchado.

Según Peña S. ${ }^{(18)}$ la especialización en la medicina no hace perder de vista la integridad y complejidad del ser humano. Sin embargo en el presente estudio, en las entrevistas realizadas se corrobora que el estudio de pacientes por órganos, muchas veces favorece la pérdida de visión en conjunto del paciente.

Sanchez D. Contreras Y. ${ }^{(19)}$ manifiesta que al efectuar la práctica de la medicina, se combina la ciencia y la tecnología, con la aplicación de conocimientos y valores, todo lo cual está presente en la relación médico-paciente, que contribuye a la solución de las necesidades del enfermo. Esto está de acuerdo con lo expresado en las entrevistas personales, efectuadas a médicos de las tres especialidades escogidos al azar.

En las últimas décadas, los cambios en la relación médico- paciente, han influenciado en el grado de satisfacción de los médicos respecto a su práctica ${ }^{(20)}$. Se ha prestado poca atención, en como los médicos experimentan y enfrentan los cambios importantes, en la práctica de la medicina ${ }^{(21)}$.

La relación médico-paciente es una oportunidad que permite al paciente expresar su agrado o desagrado, su acuerdo o desacuerdo con la atención recibida, pero también se le puede considerar como una variable, que nos permite distinguir al grupo de médicos que están satisfechos o insatisfechos con su actividad profesional ${ }^{(21,22,23)}$.

La relación médico-paciente aumenta su confianza y compromiso y produce muchos beneficios para la salud física y psicológica del paciente (Figura 2).

En conclusión, la tecnología y la especialización no contribuyen a la deshumanización del médico. El estudio de pacientes por órganos muchas veces favorece la pérdida de visión en conjunto del paciente y los plazos de espera prolongados para consulta o programación operatoria lo desaniman. El paciente necesita ser escuchado para sentirse satisfecho con su médico. La percepción de la relación médico-paciente se correlaciona fuertemente con la satisfacción del usuario y del médico. 


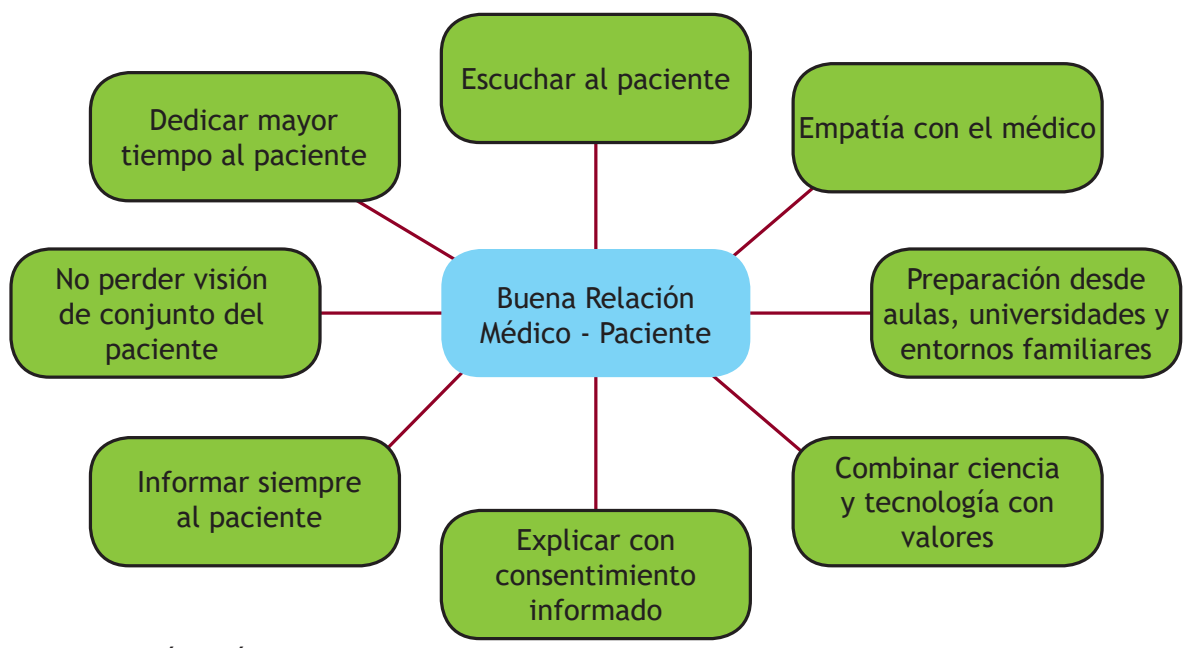

Figura 2. Buena relación médico-paciente

\section{REFERENCIAS BIBLIOGRÁFICAS}

1. González R. La Psicología en el campo de la salud y la enfermedad. La Habana: Editorial Científico Técnico; 2004.

2. Lain P. La relación médico - enfermo: Historia y teoría. Madrid: Revista de Occidente; 1994.

3. Gonzales R. La sicoterapia en nuestro medio: Un criterio y algunas experiencias. Rev Hosp Psiq. La Habana. 1984;24:167-78.

4. Rodríguez H. La relación médico - paciente. Revista Cubana de Salud Pública [Internet]. 2006 [consultado en mayo del 2017]; 32(4). Disponible en: http://scielo.sld. cu/scielo.php?pid=S0864-34662006000400007\&script $=$ sci $_{-}$ arttext\&tlng=en

5. Mendoza C. "Relación médico - paciente percibida por usuarios de consultorios externos de especialidades médicas de un Hospital nivel II del Minsa-mayo 2014" [Tesis]. Lima: Universidad San Martín de Porres; 2014.

6. Ramos C. Percepción de la relación médico - paciente, por parte de los usuarios externos de un Departamento de Medicina. An Fac med. 2008; 69(1): 12-6

7. Romero L. Percepción del paciente acerca de la calidad de atención que brinda la enfermera en el servicio de medicina en el Hospital Nacional Alcides Carrión. [Tesis]. Lima: Universidad Nacional mayor de San Marcos; 2008.

8. Fernández J. Grado de satisfacción del usuario externo en los servicios de consulta externa del Hospital Militar Geriátrico [Tesis]. Lima: Universidad Nacional Mayor de San Marcos; 2003.

9. Quix J. Actitudes y percepciones respecto de los derechos de los usuarios de los Hospitales del sector sanitario de Reus [Tesis]. Barecelona: Universidad Rovira i Virgili; 1999.

10. Somnath S. Relación médico-paciente y las disparidades raciales de la calidad del cuidado de la salud. AM J Public Health . 2003; 93:1713-9.

11. Alonso M, Kraftchenko O. La comunicación médico-paciente como parte de la formación ético-profesional de los estudiantes de medicina. Rev Cubana Educ Med Super. 2003; 17(1): 38-45.

12. Bernalte $A$. La interacción médico-paciente y sus productos. Cultura de los cuidados. 2010; 14(27): 83-9.

13. Angelucci L. Valores y factores sociodemográficos en estudiantes universitarios: Un estudio comparativo. Acta Colombiana de Psicología. 2009; 12(1):151-62.

14. Parasuman A, Zeithami V, Berry L. conceptual model of service quality and its implications for future research, Journal of marketing, 1995; 49(4):41-50.

15. Parasuman A, Zeithami V, Berry L. Alternative scales for measuring service quality: a comparative assessment based on psychometric and diagnostic criteria. Journal of marketing, 1994;70(3): 201-30

16. Biedman L, Serrano R. ¿Existe relación entre satisfacción del paciente y género del médico?. IESA Working Paper Series, 2007;2:1-25

17. Peña S. El acto médico. Cuadernos de Debate en Salud CMP. Lima: Colegio Médico del Perú; 2011

18. Sánchez D. Contreras Y. La relación médico - paciente y su importancia en la práctica médica. Rev Cubana de Medicina Militar, 2014; 43(4): 528 - 33.

19. Bascuñan M. Cambios en la relación médico - paciente y a nivel de satisfacción de los médicos. Rev Med Chile. 2005; 133(1): 11-6.

20. Zugar A. Dissatisfaction with medical practice. $\mathrm{N}$ engl J Med. 2004; 350(1): 69-75.

21. Reames H. Dunstone D. Professional Satisfaction of Physicians. Arch Intern Med. 1989; 149(9): 1951-6.

22. Lloyd M, Bor R. Communication Skills for medicine. Londres: Churchill Liviston; 2011.

23. Beck R, Daughtridge R. Sloane P. Physician - patient communication in the primary care office: A systematic Review. J am Board Fam Pract. 2002; 15(1):25-38.

\section{Fuentes de financiamiento:}

Este artículo ha sido financiado por el autor.

\section{Conflictos de interés:}

El autor declara no tener ningún conflicto de interés.

\section{Correspondencia:}

Johnny Francisco Casanova Saldarriaga

Dirección: Av. El corregidor 1531. La Molina. Lima, Perú.

Teléfono: 3652300

Correo electrónico: jfcasald@hotmail.com

\section{Recibido: 22 de agosto de 2016} Evaluado: 23 de agosto de 2016. Aprobado: 26 de junio de 2017

( ) La revista. Publicado por Universidad de San Martín de Porres, Perú. (cc) $\mathbf{B r}$ Licencia de Creative Commons Artículo en acceso abierto bajo términos de Licencia Creative Commons Atribución 4.0 Internacional. (http://creativecommons.org/licenses/by/4.0/) 